\title{
Corrigendum: Adjuvant-dependent innate and adaptive immune signatures of risk of SIVmac251 acquisition
}

Monica Vaccari, Shari N Gordon, Slim Fourati, Luca Schifanella, Namal P M Liyanage, Mark Cameron, Brandon F Keele, Xiaoying Shen, Georgia D Tomaras, Erik Billings, Mangala Rao, Amy W Chung, Karen G Dowell, Chris Bailey-Kellogg, Eric P Brown, Margaret E Ackerman, Diego A Vargas-Inchaustegui, Stephen Whitney, Melvin N Doster, Nicolo Binello, Poonam Pegu, David C Montefiori, Kathryn Foulds, David S Quinn, Mitzi Donaldson, Frank Liang, Karin Loré, Mario Roederer, Richard A Koup, Adrian McDermott, Zhong-Min Ma, Christopher J Miller, Tran B Phan, Donald N Forthal, Matthew Blackburn, Francesca Caccuri, Massimiliano Bissa, Guido Ferrari, Vaniambadi Kalyanaraman, Maria G Ferrari, DeVon Thompson, Marjorie Robert-Guroff, Silvia Ratto-Kim, Jerome H Kim, Nelson L Michael, Sanjay Phogat, Susan W Barnett, Jim Tartaglia, David Venzon, Donald M Stablein, Galit Alter, Rafick-Pierre Sekaly \& Genoveffa Franchini

Nat. Med.; doi:10.1038/nm.4105; corrected online 16 June 2016

In the version of this article initially published online, an affiliation for Luca Schifanella was omitted and there was an error in the description of the phenotypic analyses of plasmablasts in the Online Methods. The error has been corrected for the print, PDF and HTML versions of this article.

Corrigendum: Inter-individual variability and genetic influences on cytokine responses to bacteria and fungi

\author{
Yang Li, Marije Oosting, Patrick Deelen, Isis Ricaño-Ponce, Sanne Smeekens, Martin Jaeger, Vasiliki Matzaraki, Morris A Swertz, \\ Ramnik J Xavier, Lude Franke, Cisca Wijmenga, Leo A B Joosten, Vinod Kumar \& Mihai G Netea \\ Nat. Med.; doi:10.1038/nm.4139; corrected online 28 July 2016
}

In the version of this article initially published online, the url in the Online database section of the Methods was incorrect. The original version included the url http://www.bbmri.nl/molgenis/500FG. The correct url is http://hfgp.bbmri.nl/. The error has been corrected for the print, PDF and HTML versions of this article.

\section{Corrigendum: Blocking c-Met-mediated PARP1 phosphorylation enhances anti-tumor effects of PARP inhibitors}

Yi Du, Hirohito Yamaguchi, Yongkun Wei, Jennifer L Hsu, Hung-Ling Wang, Yi-Hsin Hsu, Wan-Chi Lin, Wen-Hsuan Yu, Paul G Leonard, Gilbert R Lee IV, Mei-Kuang Chen, Katsuya Nakai, Ming-Chuan Hsu, Chun-Te Chen, Ye Sun, Yun Wu, Wei-Chao Chang, Wen-Chien Huang, Chien-Liang Liu, Yuan-Ching Chang, Chung-Hsuan Chen, Morag Park, Philip Jones, Gabriel N Hortobagyi \& Mien-Chie Hung Nat. Med. 22, 194-201 (2016); published online 18 January 2016; corrected after print 25 August 2016

In the version of this article initially published, the concentrations of $\mathrm{H}_{2} \mathrm{O}_{2}$ were incorrectly labeled as micromolar ( $\mu \mathrm{M}$ ) instead of millimolar $(\mathrm{mM})$ in the legends of Figures $1 \mathrm{~h}, 3 \mathrm{c}-\mathrm{f}$ and Supplementary Figures $3,8 \mathrm{f}-\mathrm{h}, 10 \mathrm{~g}, \mathrm{j}$ and $12 \mathrm{c}, \mathrm{d}$. The error has been corrected in the HTML and PDF versions of the article.

\section{Corrigendum: High-throughput epitope discovery reveals frequent recognition of neo-antigens by CD4+ ${ }^{+}$cells in human melanoma}

Carsten Linnemann, Marit M van Buuren, Laura Bies, Els M E Verdegaal, Remko Schotte, Jorg J A Calis, Sam Behjati, Arno Velds, Henk Hilkmann, Dris el Atmioui, Marten Visser, Michael R Stratton, John B A G Haanen, Hergen Spits, Sjoerd H van der Burg \& Ton N M Schumacher

Nat. Med. 21, 81-85 (2015); published online 22 December 2014; corrected after print 18 August 2016

In the version of this article initially published, the article did not mention some restrictions on the availability of reagents. The following text has been added to the HTML and PDF versions of the paper: "The retroviral vectors containing BCL-6 and BCL-xL have been generated by a for-profit company, AIMM Therapeutics, which makes the plasmids available. Obtaining the plasmids requires an MTA (http://www.aimmtherapeutics.com/ partnering/academic-collaboration/) that includes financial obligations." The error has been corrected in the HTML and PDF versions of the article. 